\title{
Análise dos métodos de contagem de pontos e planímetro na quantificação do biofilme da dentadura - um estudo de validação metodológica $^{\dagger}$
}

\section{Analysis of the point-counting and planimetric methods in the quantification of the biofilm of dentures - a study of methodological validation}

\author{
Roseana Aparecida Gomes FERNANDES* \\ Osvaldo ZANIQUELLI** \\ Helena de Freitas Oliveira PARANHOS***
}

\begin{abstract}
FERNANDES, R. A. G.; ZANIQUELLI, O.; PARANHOS, H. de F. O. Análise dos métodos de contagem de pontos e planímetro na quantificação do biofilme da dentadura - um estudo de validação metodológica. Pesqui Odontol Bras, v. 16, n. 1, p. 63-68, jan./mar. 2002.
\end{abstract}

\begin{abstract}
Dois métodos de quantificação de biofilme da dentadura (contagem de pontos e planímetro) foram testados e comparados com o método de pesagem de papel e Índice de Higiene de Prótese. Superfícies internas de 62 próteses foram coradas, fotografadas e as áreas total e do biofilme foram projetadas em papel e contornadas com grafite. O método de contagem de pontos (experimental 1) foi aplicado com uma grade de pontos. Para o método do planímetro (experimental 2), as áreas foram medidas com um planímetro digital e para o método de pesagem (controle 1) foram recortadas e pesadas em balança de precisão. No Índice de Higiene de Prótese (controle 2), utilizou-se a atribuição de escores. Os resultados mostraram uma porcentagem de concordância entre os métodos experimentais e controle 1 de $82 \%$ (contagem de pontos) e 95\% (planímetro), bem como alto grau de correlação $(r=0,98 ; r=0,99)$ entre os valores obtidos. Quando comparados com o controle 2, houve concordância em 55\% (contagem de pontos) e 37\% (planímetro) dos casos. Os métodos experimentais podem ser úteis em estudos clínicos para avaliação da eficácia de agentes de higienização.
\end{abstract}

UNITERMOS: Prótese total; Biofilmes.

\section{INTRODUÇÃO}

A literatura mostra correlação entre má higiene e candidíase atrófica crônica. É importante o uso adequado de métodos de higienização ${ }^{1}$, bem como o conhecimento de materiais e métodos de quantificação do biofilme da dentadura, para que sejam utilizados de maneira correta e sirvam de parâmetro para testes de higienizadores ${ }^{16}$. Em Prótese Total, o método comumente preconizado baseia-se em escala de escores operacionalizada em termos de cobertura de biofilme. Evidenciadores têm sido usados combinados ou não com fotogra-fia ${ }^{11,13,14,16 \text {, }}$ 18,22,23. Além dos índices específicos para próteses totais $^{5,7,11,21,23}$, o método de pontos estimados ${ }^{3,9,15}$, microscopia eletrônica de varredura ${ }^{13}$, método espectrofluorimétrico de proteína $^{2}$, análise gráfica por computador ${ }^{14}$ e planímetro digital ${ }^{22}$ também têm sido utilizados para quantificação do biofilme.

A proposição deste trabalho foi testar dois métodos de quantificação do biofilme da dentadura: contagem de pontos (experimental 1) e planímetro (experimental 2) e compará-los com dois métodos controle: pesagem (controle 1) e Índice de Higiene de Prótese (controle 2).

\section{MATERIAL E MÉTODOS}

Os métodos foram testados em slides de estudo prévio $^{18}$. Superficies internas de 62 próteses totais superiores foram coradas (eritrosina 5\%) e fotografadas (Canon EOS Elan II) com filme para slides (ângulo de $90^{\circ}$ ). Os slides foram projetados (aumento de $10 \mathrm{X}$ ) em folha de papel e as áreas total e

†Parte da Dissertação de Mestrado.

${ }^{*}$ Mestre em Reabilitação Oral; **Professor Doutor de Materiais Dentários e Prótese; ***Professora Doutora de Prótese Total - Faculdade de Odontologia de Ribeirão Preto da Universidade de São Paulo. 
FERNANDES, R. A. G.; ZANIQUELLI, O.; PARANHOS, H. de F. O. Análise dos métodos de contagem de pontos e planímetro na quantificação do biofilme da dentadura - um estudo de validação metodológica. Pesqui Odontol Bras, v. 16, n. 1, p. 63-68, jan./mar. 2002.

do biofilme circundadas com grafite. O método de contagem de pontos (experimental 1) foi aplicado com uma grade de pontos eqüidistantes $(0,5 \mathrm{~cm}$ Page Maker versão 6.0) sobreposta ao traçado. A porcentagem de biofilme foi calculada como a relação entre $\mathrm{o} \mathrm{n}^{\circ}$ de pontos do biofilme multiplicado por 100 e o $\mathrm{n}^{\circ}$ de pontos da base interna da prótese. No método do planímetro (experimental 2), as áreas foram medidas com um planímetro digital (Placom KP 92N), com mira puntiforme sobreposta ao traçado. A porcentagem de biofilme foi calculada como a relação entre a área do biofilme multiplicado por 100 e a área total da prótese. Para o método de pesagem (controle 1), as imagens (total e biofilme) foram recortadas e pesadas em balança

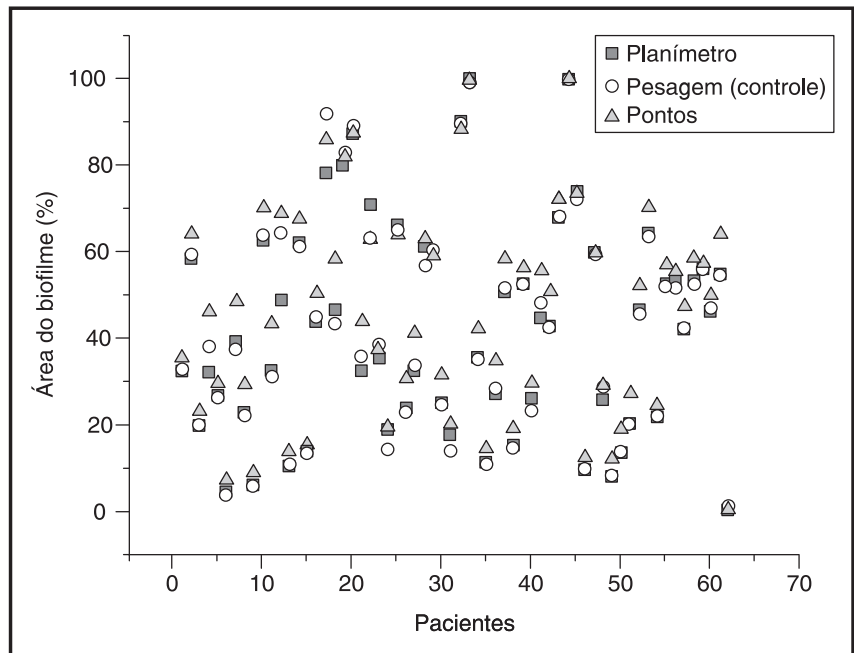

GRÁFICO 1- Porcentagem da área recoberta pelo biofilme.

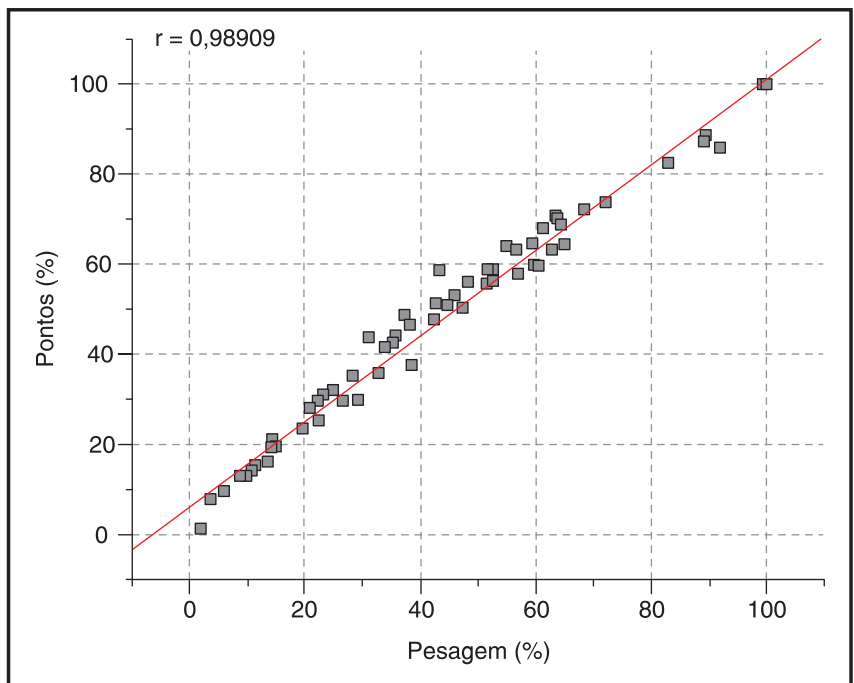

GRÁFICO 2 - Correlação entre o método de contagem de pontos (experimental 1) e pesagem (controle 1). de precisão (Metler Toledo, Switzerland). A porcentagem de biofilme foi calculada como a relação entre o peso do biofilme multiplicado por $100 \mathrm{e}$ o peso da área total da prótese.

$\mathrm{Na}$ aplicação do Índice de Higiene de Prótese ${ }^{23}$ (controle 2) cada slide foi projetado, a base da prótese dividida em 9 áreas e o acúmulo de biofilme avaliado em cada área por escores (escore 0: sem biofilme; escore 1: pontos de biofilme; escore 2: menos da metade de biofilme; escore 3: metade ou mais de biofilme; escore 4: área total de biofilme). O índice foi calculado como a relação entre o somatório dos escores individuais e o somatório das áreas avaliadas. Os escores menores que 1,5 fornecem um resultado considerado excelente; de 1,5 a 2,5 , regular e maiores que 2,5 , ruim.

\section{RESULTADOS}

O Gráfico 1 mostra os resultados (em \%) dos métodos (contagem de pontos, planímetro e pesagem), onde observamos que o controle 1 concordou em 51 casos (82\%) com contagem de pontos e em 59 casos $(95 \%)$ com planímetro. Para comparar os métodos experimentais com o controle 1, utilizou-se o teste de correlação (programa estatístico Origin). Os resultados estão apresentados nos Gráficos 2 e 3.

Para comparar os métodos experimentais e controle 1 com o controle 2, elaborou-se uma escala de escores com intervalos de classe: 1$) 0(0 \%$, escore 0 - excelente); 2) 0,01 a 1,00 (0,01 a 25\%, escore 1 - bom); 3) 1,01 a 2,00 (25,01 a $50 \%$, escore 2 regular); 4) 2,01 a 3,00 (50,01\% a $75 \%$, escore 3 -

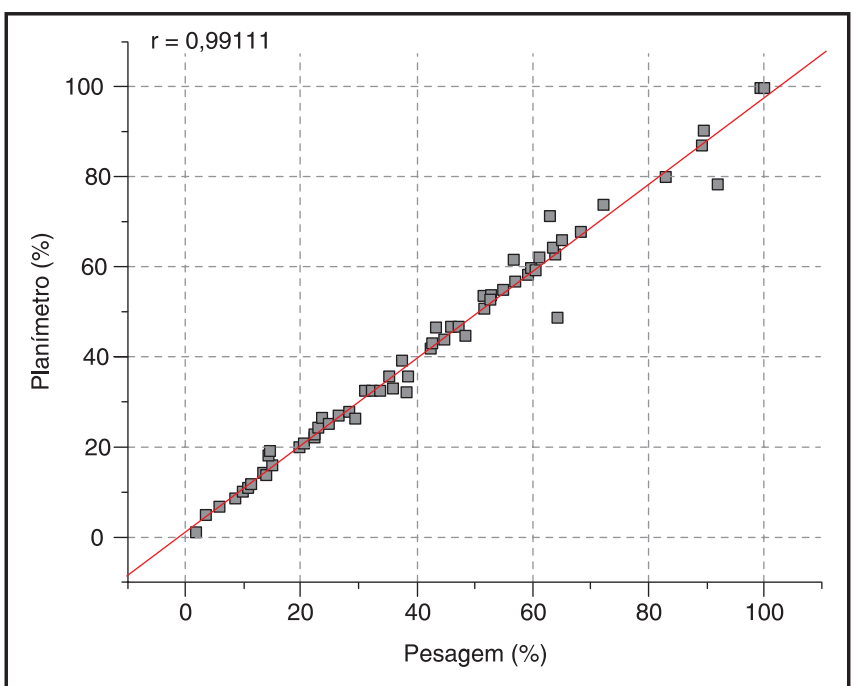

GRÁFICO 3 - Correlação entre o método do planímetro (experimental 2) e pesagem (controle 1). 
FERNANDES, R. A. G.; ZANIQUELLI, O.; PARANHOS, H. de F. O. Análise dos métodos de contagem de pontos e planímetro na quantificação do biofilme da dentadura - um estudo de validação metodológica. Pesqui Odontol Bras, v. 16, n. 1, p. 63-68, jan./mar. 2002.

ruim); 5) 3,01 a 4,00 $(75,01 \%$ a $100 \%$, escore 4 péssimo). Tal escala foi aplicada para o Índice de Higiene de Prótese (Tabela 1).

\section{DISCUSSÃO}

Trabalhos sobre quantificação do biofilme da dentadura ${ }^{1,4,16}$ chamam a atenção para importantes aspectos: a avaliação da eficácia dos higienizadores pode ser feita in vivo e in vitro, deve avaliar o biofilme residual e a capacidade de inibir o crescimento microbiano subseqüente, os materiais $\mathrm{e}$ métodos de quantificação devem ser padronizados, empregados em superfície completa da prótese e deve ser feita distinção entre os métodos que quantificam e aqueles que qualificam o biofilme. Poucos estudos têm descrito e avaliado índices de biofilme da dentadura ${ }^{4,5,7,20,23}$. A distribuição do biofilme geralmente é avaliada na superficie interna de próteses superiores ${ }^{5,7,8,11,20,23,25}$, embora a incorporação da superfície externa seja co-mum ${ }^{6,12 \text {, }}$ ${ }^{14,17-19,24}$. A fluoresceína ${ }^{17}$, eritrosina ${ }^{6,9,11}$, revelador em dois tons ${ }^{25}$, proflavina ${ }^{3,7,8}$ são os evidenciadores mais utilizados. O tipo de solução e concentração, a habilidade em corar, a facilidade de remoção e ação antimicrobiana devem ser considerados.

Nos métodos experimentais, como em estudos prévios $^{3,7-9,11,12,15,20,25}$, utilizou-se a fotografia por ser mais precisa, auxiliando a quantificação. No método visual, há necessidade de treinamento maior do observador $^{4,20}$. Todos os índices específicos para próteses totais são aplicados na superficie interna da prótese superior por meio de escores, sendo rápidos e amplamente utilizados, porém estão sujeitos a componentes subjetivos. O Índice Aditivo ${ }^{5}$ indica a inspeção do biofilme sem evidenciador e foi considerado aplicável em estudos epidemiológicos, sendo simples e confiável. O Índice de Higiene de Prótese $\mathrm{e}^{23}$ originalmente indica o evidenciador e a divisão da superfície interna em áreas, o que auxilia a atribuição de escores. Em nosso estudo, tal indice foi aplicado sobre diapositivos, facilitando a quantificação. O Índice de Budtz-Jørgensen ${ }^{7}$ (1977) tem a vantagem do uso do evidenciador, bem como da fotografia em dois ângulos e o Índice de Quigley-Hein modificado ${ }^{6,11,12,17,21,24}$ é empregado sobre superficies internas e externas, sendo a eritrosina o evidenciador geralmente utilizado.

Os métodos de contagem de pontos ${ }^{4,9,15}$ e planímetro ${ }^{22}$ mostraram-se válidos; no entanto, este exige habilidade do examinador, uma vez que a mira do aparelho deve percorrer precisamente a área a ser medida. O método de pesagem ${ }^{10,18}$ é mais traba- lhoso, porém oferece resultados precisos. O método de contagem de pontos e planímetro apresentaram, respectivamente, as maiores e menores porcentagens de biofilme (Gráfico 1). Para o método de contagem de pontos, os pontos que tangenciam a área a ser quantificada são incluídos na contagem; no método do planímetro, pequenas áreas $\left(0,1 \mathrm{~cm}^{2}\right)$ não são passiveis de medição. Porém, comparando-se os métodos experimentais com o controle 1 , não houve diferença significativa e os fatores de correlação $(r=0,98 ; r=0,99)$ foram altos (Gráficos 2 e 3), com correlação ao nível de $1 \%$. Ambos os métodos experimentais podem ser aplicados na superficie externa das próteses totais. PIETROKOVSKI et al. ${ }^{19}$ (1995) encontraram alta correlação de limpeza entre superfícies externa e interna de próteses, porém diferenças significativas entre ambas as superficies são relatadas ${ }^{6,12,24}$.

Ficou demonstrada a dificuldade em medir o biofilme por inspeção visual ${ }^{4}$, pois a análise comparativa entre os métodos experimentais e controle 2 após a transformação (Tabela 1) evidenciou diferenças significativas. Das 62 análises empregadas, 34 (55\%) concordaram com o método experimental 1 e apenas 23 (37\%) com o método experimental 2. Tal resultado é relevante, uma vez que o método controle 2 quantifica por atribuição de escores, e os métodos experimentais, por meios quantitativos. Observou-se que pacientes que receberam classificação ruim tiveram $31,3 \% ; 50,8 \%$; 68,3\%; 89,7\%; chegando até 100\% de biofilme. Pacientes com classificação excelente tiveram de 0,9\% a $15,7 \%$ de biofilme. AMBJØRNSEN et al. ${ }^{4}$ (1984), comparando métodos de atribuição de escores e contagem de pontos, encontraram superioridade deste e enfatizaram a necessidade do uso de métodos morfométricos. POULSEN et al. ${ }^{20}$ (1983), comparando dois métodos de atribuição de escores (extensão e espessura do biofilme), encontraram correlação entre ambos, porém salientam que, em estudos epidemiológicos, métodos que quantificam o biofilme podem ser usados e em experimentos de teste de higienizadores, a espessura também deve ser avaliada.

Em estudos clínicos, é importante que o examinador obtenha alto grau de consistência em seus resultados. Comparações entre diferentes examinadores são citadas ${ }^{4-6,8,11-13,20}$, porém quando é empregado um único observador, ele deve estar livre para utilizar um método mais apropriado. Em estudos epidemiológicos, o método de atribuição de escores pode ser indicado e em estudos de 
FERNANDES, R. A. G.; ZANIQUELLI, O.; PARANHOS, H. de F. O. Análise dos métodos de contagem de pontos e planímetro na quantificação do biofilme da dentadura - um estudo de validação metodológica. Pesqui Odontol Bras, v. 16, n. 1, p. 63-68, jan./mar. 2002.

TABELA 1 - Métodos de quantificação do biofilme (contagem de pontos, planímetro e pesagem) e Índice de Higiene de Prótese transformado.

\begin{tabular}{|c|c|c|c|c|c|c|c|c|c|c|c|c|c|}
\hline \multirow{3}{*}{$\begin{array}{l}\mathrm{Pa}- \\
\text { ciente }\end{array}$} & \multicolumn{6}{|c|}{ Métodos } & \multirow{3}{*}{$\begin{array}{l}\mathrm{Pa}- \\
\text { ciente }\end{array}$} & \multicolumn{6}{|c|}{ Métodos } \\
\hline & \multirow[b]{2}{*}{1} & \multirow[b]{2}{*}{2} & \multirow[b]{2}{*}{3} & \multicolumn{2}{|r|}{4} & \multirow{2}{*}{$\begin{array}{c}5 \\
\text { Índice } \\
\text { transfor- } \\
\text { mado }\end{array}$} & & \multirow[b]{2}{*}{1} & \multirow[b]{2}{*}{2} & \multirow[b]{2}{*}{3} & \multicolumn{2}{|r|}{4} & \multirow{2}{*}{$\begin{array}{c}5 \\
\text { Índice } \\
\text { transfor- } \\
\text { mado }\end{array}$} \\
\hline & & & & Escore & $\begin{array}{l}\text { Índice } \\
\text { original }\end{array}$ & & & & & & Escore & $\begin{array}{l}\text { Índice } \\
\text { original }\end{array}$ & \\
\hline 1 & 35,5 & 32,5 & 33,0 & 2,0 & Regular & Regular & 32 & 88,5 & 90,2 & 89,7 & 3,6 & Ruim & Péssimo \\
\hline 2 & 64,2 & 58,4 & 59,5 & 3,2 & Ruim & Péssimo & 33 & 100,0 & 100,0 & 99,5 & 3,7 & Ruim & Péssimo \\
\hline 3 & 23,4 & 19,9 & 20,0 & 1,8 & Regular & Regular & 34 & 42,3 & 35,7 & 35,5 & 2,4 & Regular & Ruim \\
\hline 4 & 46,2 & 32,1 & 38,2 & 3,1 & Ruim & Péssimo & 35 & 14,7 & 11,6 & 11,5 & 1,1 & Excelente & Regular \\
\hline 5 & 29,4 & 26,7 & 26,6 & 2,1 & Ruim & Ruim & 36 & 34,9 & 27,5 & 28,6 & 2,1 & Regular & Ruim \\
\hline 6 & 7,3 & 4,6 & 3,9 & 1,0 & Excelente & Bom & 37 & 58,6 & 50,8 & 51,9 & 2,8 & Ruim & Ruim \\
\hline 7 & 48,6 & 39,3 & 37,5 & 2,5 & Ruim & Ruim & 38 & 19,5 & 15,6 & 15,1 & 1,7 & Regular & Regular \\
\hline 8 & 29,4 & 23,0 & 22,3 & 2,2 & Regular & Ruim & 39 & 56,5 & 52,8 & 52,9 & 2,6 & Ruim & Ruim \\
\hline 9 & 9,3 & 6,3 & 6,3 & 1,3 & Excelente & Regular & 40 & 29,9 & 26,5 & 23,7 & 2,0 & Regular & Regular \\
\hline 10 & 70,2 & 62,8 & 63,8 & 3,3 & Ruim & Péssimo & 41 & 56,0 & 44,8 & 48,4 & 2,8 & Ruim & Ruim \\
\hline 11 & 43,4 & 32,7 & 31,3 & 2,5 & Ruim & Ruim & 42 & 51,0 & 43,0 & 42,8 & 2,6 & Ruim & Ruim \\
\hline 12 & 68,8 & 48,9 & 64,4 & 3,3 & Ruim & Ruim & 43 & 72,4 & 68,1 & 68,3 & 3,6 & Ruim & Ruim \\
\hline 13 & 13,8 & 10,8 & 11,1 & 0,7 & Excelente & Bom & 44 & 100,0 & 100,0 & 100,0 & 4,0 & Ruim & Péssimo \\
\hline 14 & 67,7 & 62,0 & 61,4 & 2,7 & Ruim & Ruim & 45 & 73,7 & 74,0 & 76,2 & 3,0 & Ruim & Ruim \\
\hline 15 & 15,7 & 14,1 & 13,7 & 0,8 & Excelente & Bom & 46 & 12,8 & 10,0 & 10,2 & 1,1 & Excelente & Regular \\
\hline 16 & 50,5 & 43,9 & 44,8 & 2,4 & Regular & Ruim & 47 & 59,9 & 60,0 & 59,8 & 2,6 & Ruim & Ruim \\
\hline 17 & 86,1 & 78,4 & 92,1 & 3,4 & Ruim & Péssimo & 48 & 29,3 & 26,4 & 29,3 & 1,6 & Regular & Regular \\
\hline 18 & 58,4 & 46,5 & 43,3 & 2,5 & Ruim & Ruim & 49 & 12,6 & 8,5 & 8,8 & 1,2 & Excelente & Regular \\
\hline 19 & 82,2 & 80,1 & 83,1 & 3,4 & Ruim & Péssimo & 50 & 19,4 & 13,8 & 14,2 & 1,4 & Regular & Regular \\
\hline 20 & 87,4 & 87,1 & 89,2 & 3,6 & Ruim & Péssimo & 51 & 27,5 & 20,63 & 20,6 & 2,0 & Regular & Regular \\
\hline 21 & 44,1 & 32,9 & 36,0 & 2,3 & Regular & Ruim & 52 & 52,7 & 46,7 & 46,1 & 2,7 & Ruim & Ruim \\
\hline 22 & 62,8 & 71,2 & 63,2 & 3,3 & Ruim & Péssimo & 53 & 70,4 & 64,3 & 63,6 & 2,6 & Ruim & Ruim \\
\hline 23 & 37,6 & 35,7 & 38,5 & 2,4 & Regular & Ruim & 54 & 25,0 & 22,1 & 22,5 & 2,0 & Regular & Regular \\
\hline 24 & 19,8 & 19,0 & 14,7 & 1,5 & Regular & Regular & 55 & 57,2 & 52,8 & 52,5 & 2,7 & Ruim & Ruim \\
\hline 25 & 64,1 & 66,3 & 65,1 & 3,2 & Ruim & Péssimo & 56 & 55,6 & 53,6 & 51,7 & 2,7 & Ruim & Ruim \\
\hline 26 & 30,9 & 24,0 & 23,0 & 2,0 & Regular & Regular & 57 & 47,5 & 41,9 & 42,4 & 2,7 & Ruim & Ruim \\
\hline 27 & 41,4 & 32,6 & 33,9 & 2,4 & Regular & Ruim & 58 & 58,7 & 53,5 & 53,0 & 2,6 & Ruim & Ruim \\
\hline 28 & 63,1 & 61,5 & 56,9 & 3,1 & Ruim & Péssimo & 59 & 57,6 & 56,6 & 57,1 & 2,8 & Ruim & Ruim \\
\hline 29 & 59,4 & 59,5 & 60,6 & 2,7 & Ruim & Ruim & 60 & 50,3 & 46,5 & 47,3 & 2,1 & Regular & Ruim \\
\hline 30 & 31,5 & 25,2 & 25,0 & 1,7 & Regular & Regular & 61 & 64,0 & 55,1 & 55,0 & 2,8 & Ruim & Ruim \\
\hline 31 & 20,7 & 18,0 & 14,4 & 1,8 & Regular & Regular & 62 & 1,0 & 0,9 & 1,0 & 0,4 & Excelente & Bom \\
\hline
\end{tabular}

1- Contagem de pontos $(\%)$ - experimental 1; 2- planímetro (\%) - experimental 2; 3-pesagem (\%) - controle 1; 4- Índice de Higiene de Prótese original - controle 2; 5- Índice de Higiene de Prótese transformado. 
FERNANDES, R. A. G.; ZANIQUELLI, O.; PARANHOS, H. de F. O. Análise dos métodos de contagem de pontos e planímetro na quantificação do biofilme da dentadura - um estudo de validação metodológica. Pesqui Odontol Bras, v. 16, n. 1, p. 63-68, jan./mar. 2002.

avaliação de higienizadores, métodos que avaliam quantitativamente o biofilme devem ser escolhidos.

\section{CONCLUSÕES}

Os métodos experimentais mostraram-se sensíveis em detectar os niveis de biofilme, podendo ser úteis em estudos clínicos para avaliar a eficácia de higienizadores. O fator de correlação entre os métodos experimentais e o método de pesagem foi alto. Houve relação próxima, mas não totalmente concordante entre as porcentagens de biofilme registradas pelos métodos experimentais e o método de atribuição de escores.

FERNANDES, R. A. G.; ZANIQUELLI, O.; PARANHOS, H. de F. O. Analysis of the point-counting and planimetric methods in the quantification of the biofilm of dentures - a study of methodological validation. Pesqui Odontol Bras, v. 16, n. 1, p. 63-68, jan./mar. 2002.

Two methods of quantification of the biofilm (point-counting and planimetric) were tested and compared with the paper-weighing method and with the Prosthesis Hygiene Index. The internal surfaces of 62 complete dentures were stained and photographed. The slides were projected on a paper sheet. The total area and the area covered with biofilm were contoured using a black pencil. The point-counting method (experimental 1) was carried out on a mesh of equidistant points. For the planimetric method (experimental 2), the areas of interest were measured by means of a digital planimeter. In the paper-weighing method (control 1) the areas of interest were cut and weighed on a precision scale. In the determination of the Prosthesis Hygiene Index (control 2), the accumulation of biofilm was estimated by means of a scoring method. The results revealed an agreement rate of $82 \%$ between the paper-weighing method and the point-counting method, and an agreement rate of $95 \%$ between the former and the planimetric method, which was confirmed by high coefficients of correlation (0.98 and 0.99, respectively). The comparison with the Prosthesis Hygiene Index resulted in 55\% of agreement with the point-counting method and in $37 \%$ of agreement with the planimetric method. The experimental methods can be useful in clinical studies involving the evaluation of the performance of denture cleansers.

UNITERMS: Denture, complete; Biofilms.

\section{REFERÊNCIAS BIBLIOGRÁFICAS}

1. ABELSON, D. C. Denture plaque and denture cleansers: review of the literature. Gerodontics, v. 1, n. 5, p. 202-206, June 1985.

2. ALTMAN, M. D.; YOST, K. G.; PITTS, G. A spectrofluorometric protein assay of plaque on dentures and of denture cleaning efficacy. J Prosthet Dent, v. 42, n. 5, p. 502-506, Nov. 1979.

3. AMBJØRNSEN, E.; RISE, J. The effect of verbal information and demonstration on denture hygiene in elderly people. Acta Odontol Scand, v. 43, n. 1, p. 19-24, Mar. 1985.

4. AMBJØRNSEN, E.; RISE, J.; HAUGEJORDEN, O. A study of examinor errors associated with measurement of denture plaque. Acta Odontol Scand, v. 42, n. 3, p. 183-191, June 1984.

5. AMBJØRNSEN, E.; VALDERHAUG, J.; NORHEIM, P. W.; FLØYSTRAND, F. Assessment of an additive index for plaque accumulation on complete maxillary dentures. Acta Odontol Scand, v. 40, n. 4, p. 203-208, June 1982.

6. AUGSBURGER, R. H.; ELAHI, J. M. Evaluation of seven proprietary denture cleansers. J Prosthet Dent, v. 47, n. 4, p. 356-359, Apr. 1982.

7. BUDTZ-JØRGENSEN, E. Prevention of denture plaque formation by enzyme denture cleanser. J Biol Buccale, v. 5, n. 3, p. 239-244, Sept. 1977.

8. BUDTZ-JØRGENSEN, E.; KELSTRUP, J.; POULSEN, S. Reduction of formation of denture plaque by a protease
(Alcalase $^{\circledR}$ ). Acta Odontol Scand, v. 41, n. 2, p. 93-98, Apr. 1983.

9. GHALICHEBAF, M.; GRASER, G. N.; ZANDER, H. A. The efficacy of denture-cleansing agents. J Prosthet Dent, v. 48, n. 5, p. 515-520, Nov. 1982.

10. ITO, I. Y.; VERRI, R. A.; RIBAS, J. P. et al. Efeitos do cloreto de cetilpiridíneo na inibição da placa dental. Odontol Mod, v. 7, n. 2, p. 8-23, fev. 1980.

11. JEGANATHAN, S.; THEAN, H. P. Y.; THONG, K. T. et al. A clinically viable index for quantifying denture plaque. Quintessence Int, v. 27, n. 8, p. 569-573, Aug. 1996.

12. KENG, S.-B.; LIM, M. Denture plaque distribution and the effectiveness of a perborate-containing denture cleanser. Quintessence Int, v. 27, n. 5, p. 341-345, May 1996.

13. KULAK, Y.; ARIKAN, A.; ALBAK, S. et al. Scanning electron microscopic examination of different cleansers: surface contaminant removal from dentures. J Oral Rehabil, v. 24, n. 24, p. 209-215, Mar. 1997.

14. MINAGI, S.; TSUNODA, T.; YOSHIDA, K.; TSURU, H. Objective testing of the efficiency of denture-cleansing agents. J Prosthet Dent, v. 58, n. 5, p. 595-598, Nov. 1987.

15. NICHOLSON, R. J.; STARK, M. M.; SCOTT, H. E. Calculus and stain removal from acrylic resin dentures. J Prosthet Dent, v. 20, n. 4, p. 326-329, Oct. 1968.

16. NIKAWA, H.; HAMADA, T.; YAMASHIRO, H.; KUMAGAI, H. A review of in vitro and in vivo methods to evaluate the 
FERNANDES, R. A. G.; ZANIQUELLI, O.; PARANHOS, H. de F. O. Análise dos métodos de contagem de pontos e planímetro na quantificação do biofilme da dentadura - um estudo de validação metodológica. Pesqui Odontol Bras, v. 16, n. 1, p. 63-68, jan./mar. 2002.

efficacy of denture cleansers. Int Prosthodont, v. 12, n. 2, p. 153-159, Mar./Apr. 1999.

17. PALENIK, C. J.; MILLER, C. H. In vitro testing of three denture-cleaning systems. J Prosthet Dent, v. 51, n. 6, p. 751-754, June 1984.

18. PARANHOS, H. F. O.; PANZERI, H.; LARA, E. H. G. et al. Capacity of denture plaque/biofilm removal and antimicrobial action of a new denture paste. Braz Dent $\mathbf{J}$, v. 11, n. 2, p. 97-104, 2000.

19. PIETROKOVSK, J.; AZUELOS, J.; TAU, S.; MOSTAVOY, R. Oral findings in elderly nursing home residents in selected countries: oral hygiene conditions and plaque accumulation on denture surfaces. J Prosthet Dent, v. 73, n. 2, p. 136-141, Feb. 1995.

20. POULSEN, S.; BUDTZ-JØRGENSEN, E.; KNUDSEN, A. M. et al. Evaluation of two methods of scoring denture plaque. Acta Odontol Scand, v. 41, n. 5, p. 283-286, Oct. 1983.

21. QUIGLEY, G. A.; HEIN, J. W. Comparative cleaning effici- ency of manual and power brushing. J Am Dent Assoc, v. 65, n. 1, p. 26-28, July 1962.

22. QUIRYNEN, M.; MARECHAL, M.; BUSSCHER, H. et al. The influence of surface characteristics on the early bacterial colonization of intra oral hard surfaces. J Clin Dent, v. 1, p. A14-19, June 1988. Suppl. A.

23. SCHUBERT, R.; SCHUBERT, U. Der ProthesenhygieneIndex (PHI). Eine methode zur dokumentation und gesundheitserziehung. Stomatol DDR, v. 29, n. 1, p. 29-31, Jan. 1979.

24. TARBET, W. J.; AXELROD, S.; MINKOFF, S.; FRATARCANGELO, P. A. Denture cleansing: a comparison of two methods. J Prosthet Dent, v. 51, n. 3, p. 322-325, Mar. 1984.

25. WALKER, D. M.; STAFFORD, G. D.; HUGGETT, R.; NEWCOMB, R. G. The treatment of denture-induced stomatitis. Br Dent J, v. 151, n. 12, p. 416-419, Dec. 1981.
Recebido para publicação em 15/05/01 Enviado para reformulação em 01/10/01 Aceito para publicação em 14/11/01

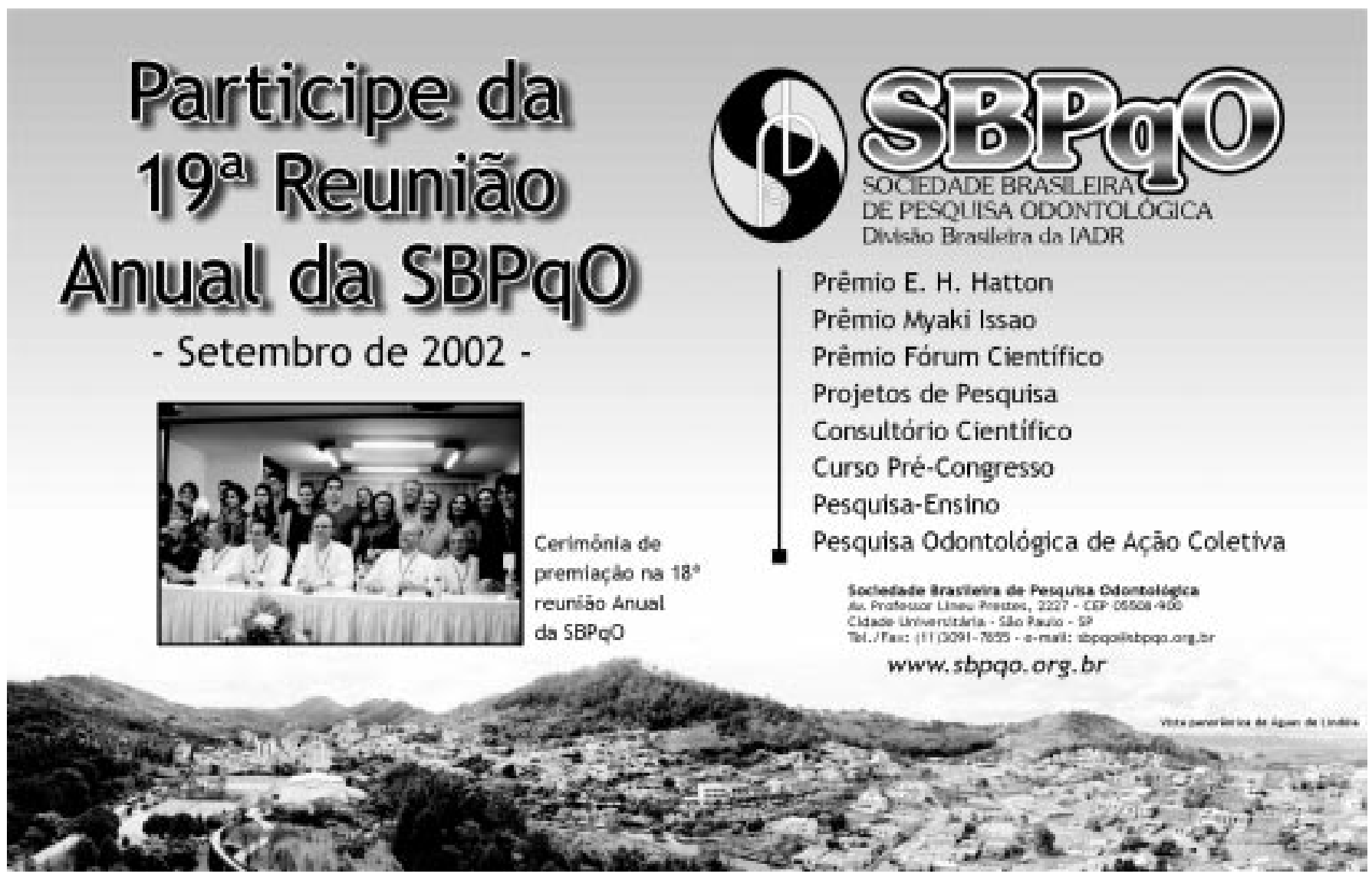

